\title{
Epigenetic Regulation of Normal and Cancer Stem Cells
}

\author{
MICHAEL F. CLARKE \\ University of Michigan Medical School, Ann Arbor, Michigan 48109, USA
}

\begin{abstract}
In many tissues, a cellular hierarchy exists in which a small population of stem cells is responsible for the production of the mature cells of the organ. The stem cells maintain themselves through a process known as selfrenewal. Similarly, tumors contain a minority population of cancer stem cells that maintain the tumor. Some factors that regulate this process of self-renewal are conserved from fruit fly to humans. Disruption of the regulation of selfrenewal results in cancer. Thus understanding the mechanisms that regulate stem cell generation has implications for normal development and disease.
\end{abstract}

KEYWORDS: self-renewal; regulation; hematopoietic stem cells; cancer

In many tissues, including the blood, gut, skin, and breast, the mature cells of a tissue are short lived and need to be replenished by stem cells. ${ }^{1-5}$ The stem cells are a minority population, and only $1 / 10,000$ cells in the bone marrow are hematopoietic stem cells (HSCs). ${ }^{1}$ The stem cells have two critical functions. First, they have the ability to differentiate into the mature cells that make up an organ. Second, during replication, stem cells can generate new stem cells through a process called selfrenewal. Self-renewal is a special type of cell division in which at least one of the daughter cells has the similar capacity to differentiate and to replicate as the original stem cell. On the other hand, progenitor cells lack the ability to self-renew. With each cell division, they become progressively more differentiated and have diminished capacity to replicate. Eventually, the progenitor cells are destined to become terminally differentiated and to stop replicating. However, a single HSC has the ability to restore blood production for the lifetime of the organism.

Notably, in the blood system, there are two populations of cells that have the ability to self-renew. The HSCs have the ability to differentiate into all of the blood elements, including red blood cells, lymphocytes, granulocytes, and platelets. ${ }^{1}$ Although their ability to differentiate is much more restricted than that of HSCs, some lymphocytes also have the ability to self-renew. ${ }^{6}$ For example, memory B cells are long-lived cells that can expand their numbers and can form mature immunoglobulin-secreting plasma cells that are short lived and are eliminated from the body after a short period of activity.

Address for correspondence: Michael F. Clarke, Professor of Medicine, University of Michigan Medical School, CCGC Room 4310, 1500 East Medical Center Drive, Ann Arbor, MI 48109. Voice: 734-764-8195; fax: 734-647-9654.

mclarke@umich.edu

Ann. N.Y. Acad. Sci. 1044: 90-93 (2005). (C) 2005 New York Academy of Sciences.

doi: 10.1196/annals.1349.012 
Cancers arise in organs that contain stem cell populations, and tumors can be viewed as a caricature of the organ from which they arise. Recent evidence demonstrates that tumors contain a minority population of cancer stem cells that drive tumor growth and metastasis. ${ }^{7-9}$ However, unlike normal stem cells whose expansion is constrained by multiple genes, cancer stem cells are constantly expanding in number, resulting in essentially unlimited tumor growth. ${ }^{10}$ Understanding the molecular control of self-renewal thus has significance for both normal organogenesis and diseases such as cancer.

To understand how self-renewal is deregulated in cancer, we began a systematic genetic analysis of normal stem cells and cancer stem cells. To do this, a cDNA library was generated from an enriched population of mouse HSCs. Then, 160,000 individual clones from this library were arrayed onto nylon membranes, which were then probed with cDNA made from mRNA obtained from mature hematopoietic cells (spleen, thymus, and bone marrow) to obtain 3,000 clones that were then used to make cDNA microarrays.

These microarrays were probed with cDNA made from HSCs and multipotent progenitor cells (MPPs). These cells differed only in their ability to self-renew; they had the same capacity to differentiate into all of the different cells of the hematopoietic lineage. From this analysis, a subset of genes was identified that was expressed at higher levels by either HSCs or MPPs. ${ }^{11}$

We chose the Bmi-1 gene for further analysis for two reasons. First, Bmi-1 is an oncogene, ${ }^{12,13}$ and we postulated that at least some oncogenes would regulate selfrenewal. Second, Bmi-1 is a member of the Polycomb family of transcriptional regulators. ${ }^{13} \mathrm{Bmi}-1$ regulates self-renewal by an epigenetic mechanism in which it suppresses the expression of a large number of target genes. Because multiple processes, such as cell survival, replication, and differentiation, are involved in self-renewal, a protein that modulates multiple genes was an attractive candidate. When analyzed at the single cell level, normal mouse HSCs, normal human HSCs, and human acute myeloid leukemia (AML) stem cells (AML tumor-initiating cells, LICs) all expressed high levels of $\mathrm{Bmi}-1 .{ }^{14}$

We found that postnatal mice deficient in the expression of Bmi-1 display failure of hematopoiesis. Bone marrow stem cells from $\mathrm{Bmi}^{-/}$mice are able to contribute to recipient hematopoiesis only transiently, indicating a primary defect in adult HSC self-renewal. This defect was specific for HSCs because normal progenitor cells were able to transiently give rise to mature myeloid blood cells. ${ }^{14}$ Subsequently, Morrison's laboratory found that Bmi-1 serves a similar function for neuronal stem cells. ${ }^{15}$

As a member of the Polycomb group family of genes, Bmi-1 functions as a transcriptional repressor and is part of a multimeric complex that interacts with DNA, leading to a repressed state of gene expression. Bmi-1 targets the INK4a locus, and overexpression of Bmi-1 results in downregulation of both p16 and p19ARF. ${ }^{16}$ Mutations of p16 resulted in a partial rescue of the Bmi-1 mutant neuronal stem cells in tissue culture. ${ }^{15}$ The situation is less clear in the hematopoietic system. Preliminary work suggests that although the INK4a locus may play some role in HSCs in normal mice, other targets downstream of Bmi-1 likely play a major role in selfrenewal of blood stem cells.

It is remarkable that cancers arise in the two self-renewing cell compartments in the hematopoietic system. Chronic myelogenous leukemia (CML), AML, and acute 
lymphocytic leukemia (ALL) are all thought to arise from HSCs or very early progenitor cells. Lymphomas arise from either B cells or T cells. These self-renewing cell compartments both require Bmi- 1 for their maintenance. ${ }^{12,14}$ Because cancers arise from cell compartments that have active self-renewal machinery, these observations suggest that acquisition of the ability to self-renew is a critical event in cancer. Note that some of this machinery is likely to still be functioning in committed progenitor cells, and mutations that reactivate other components of this pathway in these cells are also likely to be expressed by progenitor cells.

This notion is supported by the observation of Lessard and Sauvageau ${ }^{17}$ that Bmi-1 also plays a key role in malignant hematopoiesis because HOXA9/MEIS1-induced murine leukemia depends on the expression of Bmi-1. Van Lohiuzen's laboratory found that $B m i-1$ likely plays a role in some tumors of neuronal origin. Taken together with the observation that Bmi-1 is necessary for self-renewal in the blood and brain, these studies demonstrate that some components involved in the molecular regulation of self-renewal are shared by normal stem cells and cancer stem cells.

The ability to prospectively isolate cancer stem cells has implications for the study of cancer. Because the genes expressed by normal stem cells and their differentiated progeny can differ, we reasoned that the gene expression pattern of cancer stem cells and their nontumorigenic progeny could also differ. Therefore, in collaboration with Look's laboratory, we attempted to identify the genetic mutation involved in 5Q-AML. In this disease, there is a deletion of one allele of the 5Q chromosome, while the other allele is apparently normal. We reasoned that a gene(s) in the deleted region would be expressed by normal HSCs but not by their leukemic counterpart. Quantitative RT-PCR was done using mRNA isolated from normal HSCs as well as LICs. Only one of the tested genes that was expressed by HSCs was not expressed by the LICs isolated from the majority of patients with 5Q-associated AML. It is interesting that this gene is a member of a pathway implicated in the selfrenewal of Drosophila stem cells. In at least some patients, the gene is inactivated by DNA methylation. We then examined this pathway in breast cancer stem cells and found lack of expression of a member of this pathway in all five patients tested.

In summary, both normal and cancerous tissue is maintained by a stem cell population. Stem cells have the unique property of being able to generate daughter stem cells that have proliferation and differentiation capacities similar to that of the parental cell. On the other hand, progenitor cells become terminally differentiated and eventually stop proliferating. At least some of the molecular mechanisms that regulate self-renewal of normal stem cells are shared by their cancerous counterparts. However, unlike their normal counterparts, cancer stem cells have escaped the normal genetic constraints on unlimited expansion. Discovering the differences in the regulation of self-renewal of normal stem cells and cancer stem cells may give insights into new therapeutic targets.

\section{REFERENCES}

1. Morrison, S.J., N. Uchida \& I.L. Weissman. 1995. Annu. Rev. Cell Dev. Biol. 11: $35-71$.

2. Blanpain, C., W.E. Lowry, A. Geoghegan, et al. 2004. Cell 118: 635-648.

3. Alvi, A.J., H. Clayton, C. Joshi, et al. 2003. Breast Cancer Res. 5: R1-8.

4. Chepкo, G. \& G.H. Sмith. 1999. J. Mammary Gland Biol. Neoplasia 4: 35-52. 
5. Leethanakul, C., V. Patel, J. Gillespie, et al. 2000. Oncogene 19: 3220-3224.

6. Michie, C.A., A. McLean, C. Alcock \& P.C. Beverley. 1992. Nature 360: 264-265.

7. Bonnet, D. \& J. Dick. 1997. Nature Med. 3: 730-737.

8. Al-Haju, M., M.S. Wicha, A. Benito-Hernandez, et al. 2003. Proc. Natl. Acad. Sci. USA 100: 3983-3988.

9. Singh, S.K., C. Hawkins, I.D. Clarke, et al. 2004. Nature 432: 396-401.

10. Morrison, S.J., D. QIAN, L. JerebeK, et al. 2002. J. Immunol. 168: 635-642.

11. PARK, I.K., Y. HE, F. Lin, et al. 2002. Blood 99: 488-498.

12. van der Lugt, N.M., J. Domen, K. Linders, et al. 1994. Genes Dev. 8: 757-769.

13. van Lohuizen, M., M. Frasch, E. WientJens \& A. Berns. 1991. Nature 353: 353-355.

14. PARK, I.K., D. QIAN, M. KIEL, et al. 2003. Nature 423: 302-305.

15. Molofsky, A.V., R. PARdal, T. Iwashita, et al. 2003. Nature 425: 962-967.

16. JACOB, J., K. KieboOM, S. Marino, et al. 1999. Nature 397: 164-168.

17. Lessard, J. \& G. Sauvageau. 2003. Nature 423: 255-260. 